\title{
On a surface isolated by Gambier
}

Runliang Lin, Robert Conte

To cite this article: Runliang Lin, Robert Conte (2018) On a surface isolated by Gambier, Journal of Nonlinear Mathematical Physics 25:4, 509-514, DOI:

https://doi.org/10.1080/14029251.2018.1503393

To link to this article: https://doi.org/10.1080/14029251.2018.1503393

Published online: 04 January 2021 


\title{
LETTER TO THE EDITOR
}

\section{On a surface isolated by Gambier}

\author{
Runliang Lin \\ Department of mathematical sciences, Tsinghua University, Beijing 100084, P.R. China \\ RLin@mail.tsinghua.edu.cn \\ Robert Conte \\ 1. Centre de mathématiques et de leurs applications, \\ École normale supérieure de Cachan, CNRS, \\ Université Paris-Saclay, \\ 61, avenue du Président Wilson, F-94235 Cachan, France \\ 2. Department of mathematics, The University of Hong Kong, \\ Pokfulam Road, Hong Kong \\ Robert.Conte@cea.fr
}

Received 21 November 2017

Accepted 6 May 2018

\begin{abstract}
We provide a Lax pair for the surfaces of Voss and Guichard, and we show that such particular surfaces considered by Gambier are characterized by a third Painlevé function.

Keywords: Surfaces of Voss and Guichard; Lax pair; Painlevé III.

2000 Mathematics Subject Classification: 33E17, 34A05, 34Mxx.
\end{abstract}

\section{Introduction. Surfaces of Voss and Guichard}

Let us first recall two equivalent definitions of these surfaces: a geometric one and an analytic one. Our notation follows the review of Gambier [4].

Geometrically, the surfaces of Voss [11] and Guichard [6] are by definition those which admit a conjugate net made of geodesics. For instance, every minimal surface is such a surface.

Analytically, they can be characterized by their three fundamental quadratic forms dF.dF, $-\mathrm{d} \mathbf{F} . \mathrm{d} \mathbf{N}, \mathrm{d} \mathbf{N} . \mathrm{d} \mathbf{N}$, in which $\mathbf{F}(u, v)$ is the current point of the surface and $\mathbf{N}(u, v)$ a unit vector normal to the tangent plane. Choosing the coordinates $(u, v)$ defined by the geodesic conjugate net, these are [4, p. 362]

$$
\left\{\begin{array}{l}
\mathrm{I}=X_{u}^{2} \mathrm{~d} u^{2}+2 \cos (2 \omega) X_{u} Y_{v} \mathrm{~d} u \mathrm{~d} v+Y_{v}^{2} \mathrm{~d} v^{2} \\
\mathrm{II}=\sin (2 \omega)\left(X_{u} \mathrm{~d} u^{2}+Y_{v} \mathrm{~d} v^{2}\right) \\
\mathrm{III}=\mathrm{d} u^{2}+2 \cos (2 \omega) \mathrm{d} u \mathrm{~d} v+\mathrm{d} v^{2}
\end{array}\right.
$$

with the notation $X_{u}=\partial X(u, v) / \partial u, \ldots$, they depend on three functions $\omega, X, Y$ of two variables, and $2 \omega$ is the angle between the two conjugate geodesics. It is remarkable that, among the three 
Gauss-Codazzi equations [4, p. 362]

$$
\left\{\begin{array}{l}
\omega_{u v}-\frac{1}{2} \sin (2 \omega)=0 \\
X_{v}-\cos (2 \omega) Y_{v}=0 \\
Y_{u}-\cos (2 \omega) X_{u}=0
\end{array}\right.
$$

the first one characterizes the surfaces with a constant total (Gauss) curvature.

\section{Their Lax pair}

Gambier succeeded in introducing a deformation parameter $\lambda$, thus upgrading the moving frame equations to a Lax pair, but he did not write this Lax pair explicitly, so let us do it here.

The moving frame equations (Gauss-Weingarten equations) only depend on the coefficients of the first and second fundamental forms, and the spectral parameter is introduced, as in the case of surfaces with a constant mean curvature, by noticing the invariance of the Gauss-Codazzi equations (1.2) under the scaling transformation $(u, v) \rightarrow\left(\lambda u, \lambda^{-1} v\right)$. The traceless Lax pair is

$$
\begin{aligned}
& \partial_{u} \psi=L \psi, \partial_{v} \psi=M \psi, \\
& L=\left(\begin{array}{ccc}
\frac{2 X_{u u}}{3 X_{u}}+\frac{2 X_{u}}{3 X_{v}} \operatorname{cotg}(2 \omega) \omega_{v} & 0 & Y_{u} \operatorname{tg}(2 \omega) \\
-\frac{2 Y_{v}}{\lambda^{2} Y_{u}} \operatorname{cotg}(2 \omega) \omega_{u} & -\frac{X_{u u}}{3 X_{u}}-\frac{4 X_{u}}{3 X_{v}} \operatorname{cotg}(2 \omega) \omega_{v} & 0 \\
-\frac{1}{\lambda^{2} Y_{u}} \operatorname{cotg}(2 \omega) & \frac{1}{Y_{v}} \operatorname{cotg}(2 \omega) & -\frac{X_{u u}}{3 X_{u}}+\frac{2 X_{u}}{3 X_{v}} \operatorname{cotg}(2 \omega) \omega_{v}
\end{array}\right), \\
& M=\left(\begin{array}{ccc}
-\frac{Y_{v v}}{3 Y_{v}}-\frac{4 Y_{v}}{3 Y_{u}} \operatorname{cotg}(2 \omega) \omega_{u} & -\frac{2 \lambda^{2} X_{u}}{X_{v}} \operatorname{cotg}(2 \omega) \omega_{v} & 0 \\
0 & \frac{2 Y_{v v}}{3 Y_{v}}+\frac{2 Y_{v}}{3 Y_{u}} \operatorname{cotg}(2 \omega) \omega_{u} & X_{v} \operatorname{tg}(2 \omega) \\
\frac{1}{X_{u}} \operatorname{cotg}(2 \omega) & -\frac{\lambda^{2}}{X_{v}} \operatorname{cotg}(2 \omega) & -\frac{Y_{v v}}{3 Y_{v}}+\frac{2 Y_{v}}{3 Y_{u}} \operatorname{cotg}(2 \omega) \omega_{u}
\end{array}\right),
\end{aligned}
$$

with the zero-curvature condition,

$$
\left[\partial_{u}-L, \partial_{v}-M\right]=\left(\begin{array}{ccc}
-F E_{1} & E E_{1} & -\left(E G-F^{2}\right) E_{2} \\
-G E_{1} & F E_{1} & -\left(E G-F^{2}\right) E_{3} \\
G E_{2}-F E_{3}-F E_{2}+E E_{3} & 0
\end{array}\right)=0
$$

denoting $E_{j}, j=1,2,3$ the lhs of (1.2), and $E, F, G$ the coefficients of the first fundamental form,

$$
E=X_{u}^{2}, F=X_{u} Y_{v} \cos (2 \omega), G=Y_{v}^{2}
$$

\section{Surfaces applicable on a surface of revolution}

Gambier [3, p. 99] investigated surfaces whose first fundamental form I, Eq. (1.1), has coefficients $X_{u}, Y_{v}, \omega$ only depending on the single variable $x=u+v$. Denoting for shortness $X_{u}=\xi, Y_{v}=\eta$, 
he first obtains

$$
\begin{aligned}
& \mathrm{d} X=\xi \mathrm{d} u+\left(\xi+2 C_{1}\right) \mathrm{d} v, \mathrm{~d} Y=\left(\eta+2 C_{2}\right) \mathrm{d} u+\eta \mathrm{d} v, \\
& \xi+2 C_{1}=\eta \cos (2 \omega), \eta+2 C_{2}=\xi \cos (2 \omega),
\end{aligned}
$$

with $C_{1}, C_{2}$ two integration constants. After a possible conformal transformation, this defines two reductions of the Gauss-Codazzi equations, either

$$
\frac{\mathrm{d}^{2} \omega}{\mathrm{d} x^{2}}=\frac{m^{2}}{2} \sin (2 \omega), m=\text { arbitrary constant }
$$

or

$$
\frac{\mathrm{d}^{2} \omega}{\mathrm{d} x^{2}}=\frac{e^{x}}{2} \sin (2 \omega)
$$

The first reduction (3.2) integrates with elliptic functions and is handled in full detail by Gambier [3, pp. 100-105].

As to the second reduction (3.3), Gambier unexpectedly fails to integrate it. This ordinary differential equation (ODE) belongs to the class of second order first degree ODEs

$$
\frac{\mathrm{d}^{2} u}{\mathrm{~d} x^{2}}+\sum_{j=0}^{3} A_{j}(x, u)\left(\frac{\mathrm{d} u}{\mathrm{~d} x}\right)^{j}=0,
$$

whose property is to be form-invariant under the group of point transformations

$$
(u, x) \rightarrow(U, X): u=\varphi(X, U), x=\psi(X, U), U=\Phi(x, u), X=\Psi(x, u) .
$$

Roger Liouville [7] enumerated equivalence classes of (3.4) modulo the group (3.5) but, as later pointed out by Babich and Bordag [1], he forgot the important class, to which the ODE (3.3) belongs, when the invariants which he denotes $v_{5}$ and $w_{1}$ both vanish.

When $v_{5}$ and $w_{1}$ both vanish, the coefficients $A_{3}, A_{2}, A_{1}$ in the class (3.4) can be set to zero by a transformation (3.5), thus defining the five remarkable four-parameter nonautonomous ODEs

$$
\begin{aligned}
& \frac{\mathrm{d}^{2} U}{\mathrm{~d} X^{2}}=\frac{(2 \omega)^{3}}{\pi^{2}} \sum_{j=\infty, 0,1, x} \theta_{j}^{2} \wp^{\prime}\left(2 \omega U+\omega_{j}, g_{2}, g_{3}\right), \\
& \frac{\mathrm{d}^{2} U}{\mathrm{~d} X^{2}}=-2 \alpha \frac{\cosh U}{\sinh ^{3} U}-2 \beta \frac{\sinh U}{\cosh ^{3} U}-2 \gamma e^{2 X} \sinh (2 U)-\frac{1}{2} \delta e^{4 X} \sinh (4 U), \\
& \frac{\mathrm{d}^{2} U}{\mathrm{~d} X^{2}}=\frac{1}{2} e^{X}\left(\alpha e^{2 U}+\beta e^{-2 U}\right)+\frac{1}{2} e^{2 X}\left(\gamma e^{4 U}+\delta e^{-4 U}\right), \\
& \frac{\mathrm{d}^{2} U}{\mathrm{~d} X^{2}}=-\alpha U+\frac{\beta}{2 U^{3}}+\gamma\left(\frac{3}{4} U^{5}+2 X U^{3}+X^{2} U\right)+2 \delta\left(U^{3}+X U\right), \\
& \frac{\mathrm{d}^{2} U}{\mathrm{~d} X^{2}}=\delta\left(2 U^{3}+X U\right)+\gamma\left(6 U^{2}+X\right)+\beta U+\alpha,
\end{aligned}
$$

in which the summation in the first equation runs over the four half-periods $\omega_{j}$ of the Weierstrass elliptic function $\wp$.

The third one is precisely, up to rescaling, the ODE (3.3) isolated by Gambier, and the main result of Ref. [1] is the existence of a point transformation mapping these five four-parameter ODEs 
to the representation of the Painlevé equations chosen by Garnier [5], [2] (i.e. five equations with four parameters, the last one unifying $\mathrm{P}_{\mathrm{II}}$ and $\mathrm{P}_{\mathrm{I}}$ ),

$$
\begin{aligned}
\mathrm{P}_{\mathrm{VI}}: u^{\prime \prime} & =\frac{1}{2}\left[\frac{1}{u}+\frac{1}{u-1}+\frac{1}{u-x}\right] u^{\prime 2}-\left[\frac{1}{x}+\frac{1}{x-1}+\frac{1}{u-x}\right] u^{\prime} \\
& +\frac{u(u-1)(u-x)}{x^{2}(x-1)^{2}}\left[\alpha+\beta \frac{x}{u^{2}}+\gamma \frac{x-1}{(u-1)^{2}}+\delta \frac{x(x-1)}{(u-x)^{2}}\right] \\
\mathrm{P}_{\mathrm{V}}: u^{\prime \prime} & =\left[\frac{1}{2 u}+\frac{1}{u-1}\right] u^{\prime 2}-\frac{u^{\prime}}{x}+\frac{(u-1)^{2}}{x^{2}}\left[\alpha u+\frac{\beta}{u}\right]+\gamma \frac{u}{x}+\delta \frac{u(u+1)}{u-1}, \\
\mathrm{P}_{\mathrm{III}}: u^{\prime \prime} & =\frac{u^{\prime 2}}{u}-\frac{u^{\prime}}{x}+\frac{\alpha u^{2}+\gamma u^{3}}{4 x^{2}}+\frac{\beta}{4 x}+\frac{\delta}{4 u}, \\
\mathrm{P}_{\mathrm{IV}}^{\prime}: u^{\prime \prime} & =\frac{u^{\prime 2}}{2 u}+\gamma\left(\frac{3}{2} u^{3}+4 x u^{2}+2 x^{2} u\right)+4 \delta\left(u^{2}+x u\right)-2 \alpha u+\frac{\beta}{u}, \\
\mathrm{P}_{\mathrm{II}}^{\prime}: u^{\prime \prime} & =\delta\left(2 u^{3}+x u\right)+\gamma\left(6 u^{2}+x\right)+\beta u+\alpha .
\end{aligned}
$$

The point transformations which realize this mapping are, respectively,

$$
\begin{aligned}
& x=\frac{e_{3}-e_{1}}{e_{2}-e_{1}}, u=\frac{\wp\left(2 \omega U, g_{2}, g_{3}\right)-e_{1}}{e_{2}-e_{1}} \\
& x=e^{2 X}, u=\operatorname{coth}^{2} U \\
& x=e^{2 X}, u=e^{X} e^{2 U} \\
& x=X, u=U^{2} \\
& x=X, u=U .
\end{aligned}
$$

Therefore the mapping between the ODE (3.3) for $\omega(x)$ and the third Painlevé equation (3.6) for $u(\xi)$ is either

$$
e^{2 i \omega}=2 \alpha e^{-x} u, \xi=-\frac{1}{4 \alpha \beta} e^{2 x}, \alpha \beta \neq 0, \gamma=0, \delta=0,
$$

or equivalently

$$
e^{2 i \omega}=\gamma e^{-x} u^{2}, \xi=\sqrt{-\frac{1}{\gamma \delta}} e^{x}, \alpha=0, \beta=0, \gamma \delta \neq 0 .
$$

As is well known, the third Painlevé equation has three kinds of solutions:

(i) two-parameter transcendental solutions, which is the generic case, and one cannot proceed beyond the description of Gambier [3, pp. 105-106];

(ii) one-parameter Riccati-type solutions, but for our case $\gamma \delta \neq 0$ this does not happen;

(iii) zero-parameter rational ${ }^{\mathrm{a}}$ solutions, the only ones being, with the choice (3.7),

$$
u=\left(-\frac{\beta}{\alpha}\right)^{1 / 2} \xi^{1 / 2}, \gamma=0, \delta=0,
$$

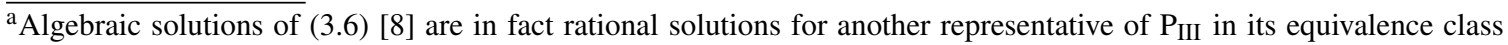
under $(u, x) \rightarrow(g(x) u, f(x))$, with $f(x)=\sqrt{x}, g(x)=1$. All algebraic solutions of $\mathrm{P}_{n}$ equations, $n=2,3,4,5$, are similarly rational.
} 
or equivalently with the choice (3.8),

$$
u=\left(-\frac{\delta}{\gamma}\right)^{1 / 4} \xi^{1 / 2}, \alpha=0, \beta=0
$$

However, these rational solutions correspond to $\sin (2 \omega)=0$, forbidden because the second fundamental form would vanish. Consequently, all solutions of (3.3) are transcendental.

\section{Future developments}

The equation (1.2) $)_{1}$ for constant total curvature surfaces (sine-Gordon equation) possesses many closed form solutions which obey neither (3.2) nor (3.3), for instance the factorized solution [10]

$$
\operatorname{tg} \frac{\omega}{2}=\frac{J_{1}(u+v)}{J_{2}(u-v)}
$$

in which $J_{1}$ and $J_{2}$ are Jacobi elliptic functions, a degeneracy of which is

$$
\operatorname{tg} \frac{\omega}{2}=\frac{\sin k(u+v)}{\sin k(u-v)}
$$

or the $N$-soliton solution [9], which depends on $2 N$ arbitrary constants. The difficulty to build VossGuichard surfaces from such solutions is the integration of the linear system $(1.2)_{2,3}$ for $X(u, v)$ and $Y(u, v)$.

Another useful development would be to find a Darboux transformation for the system (1.2).

\section{Acknowledgments}

This work was partially funded by the National Natural Science Foundation of China grant 11471182, and the Hong Kong GRF grant HKU 703313P and GRF grant 17301115. The second author also thanks the Institute of Mathematical Research (IMR), HKU for the financial support of his visit to IMR in November, 2017.

\section{References}

[1] M.V. Babich and L.A. Bordag, Projective differential geometrical structure of the Painlevé equations, J. differential equations 157 (1999) 452-485. http://doi.org/10.1006/jdeq.1999.3628

[2] R. Conte and M. Musette, The Painlevé handbook (Springer, Berlin, 2008). Russian translation Метод Пенлеве и его приложения (Regular and chaotic dynamics, Moscow, 2011).

[3] B. Gambier, Surfaces de Voss et Guichard : surfaces associées et adjointes. Déformation avec réseau conjugué permanent, Acta mathematica 51 (1928) 83-131. [Typographical errata in [4, p. 396]].

[4] B. Gambier, Surfaces de Voss-Guichard, Ann. Éc. Norm. 48 (1931) 359-396. http://www . numdam.org/item?id=ASENS_1931_3_48_-359_0

[5] R. Garnier, Sur des équations différentielles du troisième ordre dont l'intégrale générale est uniforme et sur une classe d'équations nouvelles d'ordre supérieur dont l'intégrale générale a ses points critiques fixes, Ann. Éc. Norm. 29 (1912) 1-126. http://doi.org/10.24033/asens.644

[6] C. Guichard, Recherches sur les surfaces à courbure totale constante et sur certaines surfaces qui s'y rattachent, Ann. Éc. Norm. 7 (1890) 233-264. http://archive.numdam.org/article/ASENS_1890_3_7_-_233_0.pdf

[7] R. Liouville, Sur les invariants de certaines équations différentielles et sur leurs applications, Journal de l'École polytechnique 59 (1889) 7-76.

[8] Y. Murata, Classical solutions of the third Painlevé equation, Nagoya Math. J. 139 (1995) 37-65. 
[9] A. Seeger, H. Donth and A. Kochendörfer, Theorie der Versetzungen in eindimensionalen Atomreihen III. Versetzungen, Eigenbewegungen und ihre Wechselwirkung, Z. Phys. 134 (1953) 173-193.

[10] R. Steuerwald, Über Enneper'sche Flächen und Bäcklund'sche Transformation, Abh. Bayer. Akad. Wiss. (München) 40 (1936) 1-105.

[11] A. Voss, Über diejenigen Flächen, auf denen zwei Scharen geodätischer Linien ein conjugirtes System bilden, Sitzungsberichte der Königl. Bayerischen Akademie der Wissenschaften zu München 18 (1888) $95-102$. 